\title{
Débat pourtour méditerranéen
}

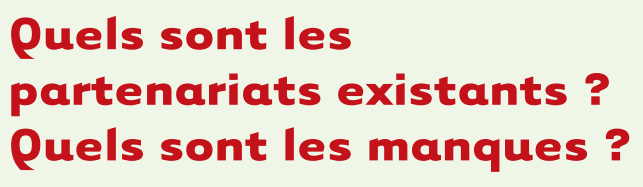

Quels sont les

partenariats existants?

Quels sont les manques?

L'organisation de la prise en charge des maladies rares constitue un modèle pour beaucoup, même si de nombreuses améliorations doivent encore être apportées. J'ai toujours un très grand plaisir à accueillir mes amis de l'autre côté de la Méditerranée.

Marie-Françoise Bendridi, Hôpital de la Rabta à Tunis Pays maghrébin de 12 millions d'habitants, la Tunisie fait partie des 23 pays du pourtour de la Méditerranée. Elle a été et reste sillonnée par de nombreuses populations. Elle présente un taux de consanguinité élevé (32\%), qui peut atteindre $90 \%$ dans certains groupes de pathologies. Cela explique pourquoi les maladies ne sont pas si rares, mais sont plutôt orphelines.

L'intérêt pour les maladies héréditaires du métabolisme est né en 1987 lorsque les pédiatres, confrontés à des maladies familiales et des décès précoces liés à des encéphalopathies et des handicaps moteurs, se posaient des questions. Ils recevaient des laboratoires européens des diagnostics positifs de maladies héréditaires du métabolisme. Comme le laboratoire de biochimie de l'hôpital de la Rabta disposait d'un chromatographe, une unité bioclinique composée de pédiatres et de biochimistes a été constituée. En outre, un projet franco-tunisien a été mis en place sur le dépistage orienté des maladies héréditaires du métabolisme.

À partir de 1987, les priorités suivantes ont été définies:

\section{- l'estimation de la fréquence}

En Tunisie, les cas de pathologies métaboliques sont nombreux. On observe en outre une grande fréquence de la phénylcétonurie (PCU), une prédominance par rapport aux fréquences mondiales de syndromes de Morquio et de Maroteaux-Lamy, beaucoup de maladies de Niemann-Pick de type B, et 105 cas de maladie de Gaucher (avec une particularité de prépondérance par rapport aux séries internationales de type 2 et 3 ).

\section{- la formation de professionnels spécialisés}

Dans ce domaine, nous avons eu le concours de l'équipe du Dr J.-M. Saudubray, puis de l'équipe du Dr H. Ogier. Je remercie grandement cette dernière, car elle a formé les six pédiatres du centre de maladies

métaboliques de la Rabta, ainsi que les nutritionnistes et la psychologue, auxquels se sont ajoutés la biologiste, l'assistante sociale et les kinésithérapeutes. Nous voyons maintenant que les neuropédiatres et les internistes commencent à s'intéresser aux maladies métaboliques. Ces formations nous ont permis de confirmer un nombre croissant de cas. Entre 1987 et 2012, une quarantaine de cas par an ont été confirmés, notamment grâce à des projets de coopération.

- la sensibilisation des médecins et des autorités au dépistage et à la prise en charge précoce

Pour sensibiliser les médecins et les autorités, nous avons mis en place des journées PCU et des écoles métaboliques, auxquelles ont participé un grand nombre de confrères tunisiens et belges. L'association des maladies métaboliques a été créée et organise ces journées. Depuis quelques années, les familles participent à ces journées et à ces écoles.

\section{- le développement de la recherche}

En 2004, nous avions sept unités de recherche, qui ont pu à la fois développer le registre national de la maladie de Gaucher et participer à des projets de recherche fédérés et nationaux sur le polyhandicap et le retard mental. En outre, nous sommes centre d'essai depuis 2008.

\section{- le développement du plateau technique}

Initialement, le laboratoire de biochimie de la Rabta disposait d'un chromatographe d'acides aminés à couche mince. II a ensuite 
été équipé d'un analyseur d'acides aminés et d'un fluorimètre, qui nous ont permis de réaliser un grand nombre de diagnostics. Malheureusement, l'analyseur est tombé en panne. Nous avons donc dû réutiliser le chromatographe d'acides aminés à couche mince. Le laboratoire dispose en outre d'un UPLC (chromatographe liquide ultra performance), mais il ne résout pas l'ensemble des problèmes que nous rencontrons. Parmi les examens que nous sommes capables d'effectuer figure la glycogénose ; nous réalisons désormais le diagnostic en Tunisie. La plupart des examens sur le métabolisme intermédiaire se font à Tunis. Le centre de Sousse est quant à lui en train de se spécialiser sur les examens liés aux maladies de surcharge.

\section{- la prise en charge des malades}

Pour les maladies du métabolisme intermédiaire, nous traitons 80 malades atteints de glycogénose I et III, ainsi que les aminoacidopathies et les aciduries organiques. Depuis 1999, les mixtures d'acide aminé sont remboursées. En matière de régime, nos diététiciennes ont été très créatives pour tenir compte du fait que les farines hypoprotidiques ne sont pas prises en charge par la Caisse de sécurité sociale. Avec la Maïzena, nous avons conçu un livre de recettes; une mère a même créé un couscous à base d'amidon - j'espère qu'elle obtiendra son brevet d'invention d'ici peu de temps. Nous avons par ailleurs réalisé des livrets éducatifs et des films sur la PCU et la tyrosinémie. Nous organisons régulièrement des ateliers locatifs pour les familles, en Tunisie, mais aussi en Algérie. Sur 187 malades PCU diagnostiqués, nous n'en traitons que 62 (17 à la naissance et 45 entre 6 mois et 6 ans et demi). Depuis 2001, nous disposons du médicament NTBC ([2-(2-nitro-4-trifluoromethylbenzoyl)1,3-cyclohexanedione]), qui nous permet de traiter 24 malades sur 60 diagnostiqués. Les maladies de surcharge lysosomale restent le parent pauvre, en dehors de la cystinose. Nous sommes obligés d'avoir recours à la greffe de moelle osseuse. Depuis quelques années, nous réalisons des greffes de moelle osseuse pour enfants. Aujourd'hui, nous avons l'expérience de 6 patients. Nous effectuons des essais cliniques dans le cadre d'un contrat particulier qui nous permet de poursuivre le traitement des malades à l'issue de l'essai clinique; 15 malades vont ainsi pouvoir être traités ( 8 d'entre eux sont pour l'instant sous post-essai clinique).

Les avancées réalisées entre 1987 et 2013 sont le fruit de partenariats nationaux - avec des laboratoires d'investigation tunisiens, tels que I'Institut Pasteur, le laboratoire de Sousse, les services spécialisés tunisiens - et internationaux. S'agissant des laboratoires d'investigation étrangers, je remercie spécialement Mesdames Poenaru, Caillaud et Vanier qui nous ont beaucoup aidés pour la confirmation diagnostique des malades. Les sociétés pharmaceutiques nous ont, quant à elles, aidés en matière de formation. Depuis de nombreuses années, nous participons aux manifestations internationales. II existe désormais une association de parents en Tunisie. En outre, nous avons conclu un partenariat avec l'association «Les Feux Follets » pour le métabolisme intermédiaire, et un partenariat est en cours d'élaboration avec l'association VML (Vaincre les maladies lysosomales) France. Le chemin parcouru était ardu; le chemin à parcourir sera peut-être meilleur. Parmi nos points forts figure le fait que nous avons réalisé des études multicentriques et disposons d'un registre national de la maladie de Gaucher. Ce dernier ne suffit toutefois pas à estimer la fréquence, compte tenu de l'absence de dépistage systématique néonatal qui devait être mis en place en décembre 2010. Depuis la révolution de janvier 2011, nous sommes dans une période transitoire qui ne facilite pas l'instauration d'un dépistage systématique. En outre, il existe une sous-estimation par insuffisance de diagnostic.

Malgré tout, nous constituons un centre de référence pour le Maghreb. Le laboratoire spécialisé de la Rabta et l'expertise clinique du service de pédiatrie de la Rabta font que tous les pédiatres envoient les malades à notre centre. En outre, nous recevons des malades de pays voisins (Algérie, Libye, Mauritanie et Sénégal). Par ailleurs, nous avons pu dresser l'histoire naturelle de la maladie de Niemann-Pick B et de la maladie de Gaucher, et établir le spectre mutationnel et - peut-être les faits fondateurs de certaines maladies.

Cependant, notre plateau technique est insuffisant puisque nous ne disposons que d'un seul laboratoire spécialisé qui assure parallèlement la routine d'un hôpital. De plus, nous regrettons beaucoup que les techniques développées dans le cadre de la recherche partent avec le chercheur. En outre, nous constatons que la crise économique a pratiquement épuisé l'aide du partenariat bénévole européen.

Concernant la prise en charge, l'accessibilité au traitement pour certaines maladies s'est améliorée. Nous avons contextualisé nos régimes spécifiques. Nous avons donné l'accessibilité au diagnostic anténatal. Nous avons choisi des alternatives thérapeutiques, comme la greffe de moelle. Nous avons commencé à sensibiliser au diagnostic et à la prise en charge en Tunisie et au Maghreb. II n'en demeure pas moins que les diagnostics et les traitements sont tardifs. Certains traitements spécifiques et coûteux nous font défaut. Des difficultés dans la réhabilitation existent. Des problèmes éthiques se posent pour la greffe de moelle osseuse. Enfin, un réseau de diagnostic et de prise en charge national ou maghrébin reste à créer.

Cette situation génère une certaine frustration de la part des parents et du personnel soignant, malgré la présence d'associations. Sur le plan de la recherche, nous disposons certes d'un laboratoire de recherche, mais ses moyens financiers sont limités.

Les perspectives suivantes ont été identifiées :

- mettre en place le dépistage systématique néonatal pour les maladies traitables (hypothyroïdie et PCU) ;

- diagnostiquer précocement les maladies les plus fréquentes et traitables (ce qui implique l'accès à un plateau technique opérationnel et le développement des compétences en pédiatrie, néonatologie, neurologie, radiologie, etc.) ; 
- traiter précocement et régulièrement : cela implique une accessibilité pour tous les malades, une accessibilité aux thérapeutiques spécifiques (grâce à des prix préférentiels pour la Tunisie et une fabrication industrielle locale), une accessibilité aux régimes (levée des taxes douanières pour les produits hypoprotidiques), la mise à disposition d'un appareil d'hémofiltration, une rééducation optimale pour tous les malades, et la mise en place d'une consultation multidisciplinaire; - mettre en place un réseau de diagnostic et de prise en charge pour éviter les doublons dans la prise en charge : ce réseau devrait d'abord concerner les diagnostics tunisiens, puis maghrébins, voire européens - pour ce que nous ne pourrions pas faire au Maghreb;

- développer des registres nationaux, qui sont très importants pour la fiabilité clinique et l'épidémiologie ;

- renforcer le partenariat associatif du point de vue scientifique et du point de vue des associations de parents.

\section{Rachida Boukari, Présidente de la Société algérienne de pédiatrie} Pour réaliser cette présentation sur la mucoviscidose en Algérie, j'ai travaillé en collaboration avec des collègues pédiatres de certaines régions du pays.

\section{Introduction}

La mucoviscidose est une maladie rare. Son incidence est diversement appréciée en fonction des pays. En 2011, 6000 malades étaient recensés en France. La prévalence est aussi élevée en Algérie et dans d'autres pays du Maghreb. Pendant de très longues années, il a été dit que cette maladie n'existait pas en Algérie - ce qui a très certainement freiné le diagnostic de cette affection. Aujourd'hui, l'incidence de cette maladie n'est pas connue de manière précise, car nous n'avons pas de données épidémiologiques, ni de recensement, ni de notification précise, ni de registre de cette maladie. Le nombre de cas recensés correspond aux cas colligés dans les services hospitaliers de la capitale et des grandes villes d'Algérie.

Pour une population de 38 millions d'habitants et une cohorte néonatale de près d'un million de personnes, nous estimons le nombre de nouveaux cas à près de 250 par an. L'immense majorité d'entre eux n'est pas diagnostiquée. Les ressources et dépenses de santé dans notre pays sont encore très faibles.

La mucoviscidose est une maladie à transmission autosomique récessive. Compte tenu du taux de consanguinité élevé au Maghreb - il atteint presque $25 \%$ en Algérie - il fait peu de doute que cette maladie est plus fréquente qu'on ne le pense, et au moins aussi fréquente qu'en France. Notre retard est donc réel en matière de diagnostic.

\section{État des lieux de la prise en charge de la mucoviscidose en Algérie}

La mucoviscidose est encore mal connue en Algérie. Le diagnostic de cette maladie a été pendant longtemps freiné par le fait que le principal outil de diagnostic, le test de la sueur, n'était disponible que dans une seule région.

Entre 1997 et 2000 , le nombre de cas était faible. À partir de la mise en place d'un début de réseau en 2005 , le nombre de cas diagnostiqués a progressé - même s'il reste encore largement en deçà de la réalité.
Les patients sont principalement pris en charge à Alger et Oran. La sous-évaluation des cas s'explique par les difficultés d'accessibilité, puisque la majorité des patients doit se rendre à Alger. Seules les formes les plus sévères de la maladie sont diagnostiquées, car le délai au diagnostic est important (deux à trois ans en moyenne). En outre, l'âge de diagnostic est généralement de 3 ans et plus. La consanguinité des cas colligés atteint $55 \%$; le nombre de décès dans la fratrie s'élève à $46 \%$. Seul le phénotype clinique le plus sévère est observé et diagnostiqué [symptomatologie respiratoire chronique fréquente dans $65 \%$ des cas; hypotrophie (63\%) ; diarrhée chronique et déshydratation aiguë (83\%) ; toux chronique et encombrement (63\%)].

\section{Les actions réalisées et les partenariats actuels}

Pour commencer à améliorer l'accès au diagnostic et aux soins, nous avons d'abord ciblé l'action de formation et de sensibilisation des pédiatres et des médecins généralistes. Un premier cycle de formation a donc été organisé pour porter à la connaissance des médecins que la mucoviscidose existait bel et bien en Algérie et qu'un diagnostic devait absolument être réalisé. Le Pr Gabriel Bellon, qui est le responsable du service de pédiatrie du Centre de ressources et de compétence de la mucoviscidose de Lyon, était à nos côtés pour mener ces actions. Entre 2009 et 2013 , il a sillonné l'Algérie avec les pédiatres algériens dans le cadre de rencontres scientifiques régionales, nationales et maghrébines visant à sensibiliser et former des pédiatres. II a aussi souvent effectué des visites dans le service pour assurer l'expertise médicale auprès de certains patients. Ainsi je le remercie tout particulièrement de ce partenariat informel, qui va se poursuivre.

L'accès au test de la sueur, qui constitue la clé du diagnostic, était le problème fondamental. II est donc apparu très rapidement comme indispensable de multiplier le nombre de laboratoires capables de réaliser ce dosage du chlore sudoral. Formé par le Pr Bellon, le Dr Radoui a assuré une formation en cascade dans différents centres, avec le soutien des laboratoires Erempharma qui ont fourni le matériel pour le test de la sueur. II était important que celui-ci soit fait à partir de la technique de référence. Aujourd'hui, ce test est réalisé à Alger, à Oran, à Constantine et dans plusieurs régions de l'Ouest du pays. En 2014, d'autres laboratoires seront capables de réaliser ce test.

Sur le plan thérapeutique, la prise en charge respiratoire se fait, non pas dans des centres de référence (puisque l'Algérie n'en compte aucun), mais de façon éparpillée dans les services de pédiatrie, essentiellement sous forme d'antibiothérapie par voie intraveineuse. L'accès 
aux médicaments efficaces sur le Pseudomonas aeruginosa demeure difficile. Nous essayons d'utiliser les protocoles et les recommandations donnés pour traiter ces infections. La kinésithérapie constitue notre cheval de bataille, car elle pose de vrais problèmes. Depuis septembre 2013, un cycle de formation à la kinésithérapie respiratoire a été mis en place avec le Pr Bellon et l'équipe de l'Institut des sciences et techniques de la réadaptation de Lyon. Le premier séminaire a eu lieu en septembre 2013 à Alger; d'autres séminaires sont programmés dans plusieurs régions du pays. Leur but est de former à chaque session une trentaine de kinésithérapeutes spécialisés dans la kinésithérapie respiratoire de l'enfant.

Pour la prise en charge digestive et nutritionnelle, nous avons également rencontré de réelles difficultés. L'utilisation des extraits pancréatiques a apporté un plus fondamental dans la prise en charge digestive des enfants atteints de mucoviscidose. Elle n'est remboursée par la Sécurité sociale que depuis 2013.

Nous n'avons aucune participation dans des projets de thérapie génique, ni de thérapie protéique. Nous avons réussi à améliorer l'accès aux soins, mais beaucoup de travail reste à faire. En 2007, le réseau mucoviscidose, qui vise à faire travailler ensemble un certain nombre de pédiatres, s'est constitué à Alger. Depuis, d'autres régions du pays ont rejoint le réseau, qui a permis de nouer des partenariats avec des sociétés savantes de pédiatrie et de pneumologie pédiatrique, avec l'association Vaincre la Mucoviscidose, la Société française de la mucoviscidose et le laboratoire Erempharma.

Pendant longtemps, il n'était pas question de réaliser l'étude génotypique puisque l'urgence était pour nous de traiter les formes les plus sévères. Avec le développement possible de la thérapie génique, l'identification des génotypes peut être utile si nous réalisons dans un avenir proche un dépistage ciblé, car il n'est pas question de prendre simplement les mutations identifiées dans les autres pays. Sur une série de 38 enfants et de 70 parents (soit 146 allèles), nous avons pu nous faire une idée des mutations présentes en Algérie. Nous avons ainsi pu voir que la fréquence de la mutation delta $\mathrm{F} 508$ représente $23 \%$ des allèles, contre $68 \%$ en France. Certaines mutations sont plus fréquentes en Algérie, alors qu'elles n'existent presque pas en France. La connaissance de la répartition des mutations sera très utile dans l'utilisation future d'un kit de dépistage.

\section{Nos besoins et attentes}

Notre action de sensibilisation, de formation et d'enseignement va se poursuivre. En outre, nous allons poursuivre et étendre l'implantation du test de la sueur dans les laboratoires. L'amélioration de la prise en charge et l'accès aux médicaments devra se poursuivre. Les thérapeutiques nébulisées vont être développées; plusieurs ateliers de formation ont été organisés sur la nébulisation. Pour être convaincant vis-à-vis de nos autorités, il est fondamental qu'un registre national soit mis en place. Cet outil permettra notamment de planifier les soins, et d'identifier et suivre les patients. Le partage d'expérience et l'accompagnement sont essentiels à la mise en place de ce registre. $\varepsilon$ n matière d'organisation des soins, la mise en place de centres d'expertise et de centres de référence sera fondamentale. Sur ce sujet, nous nous inspirerons des expériences française et tunisienne sur le registre des maladies métaboliques. En outre, nous nous efforcerons de développer le diagnostic moléculaire afin de réellement connaître et identifier les mutations les plus fréquentes. Dans ce domaine, notre besoin de coopération est réel, afin notamment que nos généticiens soient formés et que des laboratoires de génétique soient mis en place.

Le dépistage néonatal de la mucoviscidose pourrait, dans un premier temps, se faire de manière ciblée pour obtenir une appréciation du taux d'incidence dans notre pays. Ensuite ce dépistage pourrait être élargi à toute la cohorte néonatale.

\section{Brigitte Chabrol}

Hicham Mansour, que nous allons maintenant écouter, est libanais. Il a suivi une formation dans les maladies rares et les maladies du métabolisme à Marseille pendant deux ans. Il va nous montrer ce qu'il a mis en place depuis son retour au Liban en 2009.

\section{Hicham Mansour, Liban}

Le Liban s'étend sur $10000 \mathrm{~km}^{2}$ et compte 4,8 millions d'habitants. $10 \%$ d'entre eux sont porteurs de «Carte de personne avec Handicap ». On dénombre dans le pays environ 440000 réfugiés palestiniens, 1,2 million de réfugiés syriens et 100000 réfugiés irakiens. La présence de ces réfugiés représente un poids conséquent pour le ministère de la Santé et le ministère des Affaires sociales.

Le Liban ne dispose d'aucun registre national pour les maladies neuromusculaires. En outre, le taux de consanguinité est globalement élevé dans le pays; seule la région urbaine de Beyrouth présente un taux plus faible. Dans de nombreuses régions, des cas de maladies mitochondriales ont été recensés. Sur 110 patients atteints de maladies mitochondriales diagnostiqués en quatre ans, le taux de consanguinité s'élève à $59 \%$. Par ailleurs, j'ai recensé 51 cas de maladies neuromusculaires, dont 18 avaient des myopathies mitochondriales pures.

Au Liban, la médecine est majoritairement privée. On compte actuellement dix neuropédiatres (dont huit à Beyrouth), deux généticiens et un laboratoire d'anatomopathologie qui est capable de réaliser des études sur une biopsie neuromusculaire. Le diagnostic est un examen très cher que le patient doit payer. Le coût de la prise en charge n'est également pas négligeable (20 minutes de physiothérapie coûtent 20 \$ pour un salaire minimal de $580 \$$ par mois). Le secteur privé est très bien équipé, mais ses prestations sont très coûteuses. Une solution consiste donc à orienter les 
patients vers le ministère des Affaires sociales en présentant leur « carte de handicap ». Comme cette expression n'est pas encore très bien acceptée par la société libanaise, les patients refusent souvent cette carte. Ils sont alors perdus de vue pendant un certain temps, avant de réaliser qu'ils ne peuvent pas être pris en charge sans cette carte du ministère.

Le travail législatif sur le handicap a débuté en 1973. La période de guerre que le Liban a connu entre 1975 et 1991 a retardé la mise en place de cette législation. Un essai de prise en charge conditionnelle des patients handicapés a été effectué en 1983, mais ce n'est qu'en 1993 qu'un vrai travail sur le droit des patients handicapés a été initié. Depuis une loi adoptée en 2000 , le patient peut recevoir une «carte de handicap » qui lui donne le droit d'accéder, grâce - en théorie - à une prise en charge totale de l'État, à tous les services de santé clinique et paraclinique, ainsi qu'aux services de réadaptation et de soutien assurés dans les établissements publics et privés. Comme le ministère des Affaires sociales et le ministère de la Santé doivent s'occuper des Libanais et des réfugiés, le secteur privé et les $0 N G$ sont souvent sollicités.

Le Liban compte plusieurs confessions religieuses qui ont chacune essayé de créer leurs propres associations. Certaines sont capables de traiter les maladies neuromusculaires, tandis que d'autres, de taille plus mineure, gèrent uniquement la physiothérapie ou l'orthophonie. L'association Sesobel, l'Organisation des handicapés, le Caritas, l'Arcen-Ciel et l'Al-Kafaat sont capables de gérer un enfant atteint de polyhandicap et d'une maladie neuromusculaire.

Les enfants malades n'ont pas tous la possibilité d'aller à Beyrouth ; nous avons donc décidé d'aller chez eux. Des organisations sont prêtes à créer des établissements et à financer les orthophonistes, les psychomotriciens, les psychologues, etc. Un médecin est nécessaire pour couvrir les différentes régions. Je citerai les exemples de deux associations qui sont les seules capables au Liban de gérer les patients atteints de maladies neuromusculaires - en réalité, 60 \% d'entre eux sont couverts.

Fondée en 1976 par Yvonne Chami, l'association Sesobel (Service social pour le bien-être de l'enfant - Liban) prend en charge 655 enfants - libanais à $96 \%$ - dont neuf sont atteints de la maladie de Duchenne, deux de Marinesco-Sjögren, un de neuropathie à axones géants, deux d'amyotrophie spinale, un de leucodystrophie métachromatique, trois de myopathie congénitale, sept de myopathie mitochondriale, deux d'ataxie de Friedreich et deux d'ataxie-télangiectasie.

Le Sesobel réunit des médecins volontaires et une équipe de travail composée de psychomotriciens, d'orthophonistes, de diététiciennes et de physiothérapeutes. Le Pr Joseph Haddad, qui est l'un des premiers à avoir travaillé à la mise en place d'une organisation de la prise en charge des maladies neuromusculaires, dirige l'équipe médicale qui se compose de deux neuropédiatres, d'un cardiopédiatre, de deux gastro-entérologues, d'un généticien, d'un pneumopédiatre, d'un ORL, de trois orthopédistes pédiatres et d'un neurochirurgien. Cette équipe prend en charge tous les patients de l'établissement.

L'unité de physiothérapie assure trois sessions par semaine. Comme elle ne peut pas offrir davantage de séances, une éducation parentale est assurée pour que les parents apprennent à soigner leurs enfants à domicile (à raison d'une demi-heure par jour). L'unité d'orthophonie assure une prise en charge neuro-alimentaire et de communication de trois séances par semaine. L'unité d'ergothérapie propose, quant à elle, une séance par semaine; une éducation parentale est également dispensée pour assurer la continuité des soins à domicile. Des aides techniques sont par ailleurs disponibles. Le Sesobel est le seul établissement au Liban à disposer d'un laboratoire d'étude de la marche et du mouvement. II propose des aides techniques de posture, des aides techniques de mobilité, des aides techniques pour l'activité de vie journalière, ainsi que des aides techniques pour l'aménagement des lieux de vie.

Le Sesobel a conclu de nombreux partenariats en France (association Partage, association Terre de Vie, association française contre les myopathies), mais aussi aux États-Unis, en Australie et en Arabie Saoudite. Ces partenaires aident le Sesobel à poursuivre son action, malgré les difficultés financières qu'il rencontre. Celles-ci s'accompagnent d'un manque de personnel et d'un manque de suivi des patients de plus de six ans, dont la plupart sera orientée vers une association sœur «Anta Akhi ».

La seconde association que je souhaite vous présenter est la Lebanese Welfare Association for the handicapped, qui dispose d'un centre médical de jour et d'une école spécialisée. Cet établissement, qui gère deux sites majeurs bien équipés dans la région sud et la Bekaa, est capable de diagnostiquer un certain nombre de patients. Néanmoins, je n'ai pas pu trouver les moyens financiers nécessaires à la poursuite de mon diagnostic pour une centaine d'enfants atteints de maladies neuromusculaires.

L'équipe de travail de cette association se compose de deux neuropédiatres, d'un médecin de réadaptation, de deux orthopédistes, d'un neurochirurgien, de psychomotriciens, d'orthophonistes, de diététiciennes et de physiothérapeutes.

La prise en charge est assurée en physiothérapie, en orthophonie et en ergothérapie. En outre, diverses aides techniques sont assurées, notamment pour réaliser des orthèses. L'établissement est très bien équipé - en psychomotricité, en orthophonie, en stimulation neurosensorielle, etc. - mais le personnel présent n'est en mesure de le faire fonctionner qu'à 20 ou $30 \%$ de ses capacités.

Le suivi des patients n'est pas encore optimal. Ils peuvent être suivis pendant quelques mois, puis sont perdus de vue. En outre, l'association ne propose pas de prise en charge respiratoire pour les patients atteints 
de maladies neuromusculaires. Le diagnostic s'arrête donc à une certaine étape chez ces patients.

La Lebanese Welfare Association for the handicapped a conclu uniquement des partenariats nationaux.

Les principaux manques et difficultés rencontrés au Liban, en particulier dans le domaine des maladies neuromusculaires, portent sur le financement (du diagnostic ou de la prise en charge), l'éducation sociale (en arabe, le terme « handicap » reste toujours peu utilisé, car il sous-tend que la personne concernée est incapable de faire plusieurs choses), la clarification et l'application des lois et des droits des patients, et l'organisation du corps médical - qui reste très centralisé à Beyrouth.

Parmi les solutions possibles figurent la création d'un registre national, une meilleure répartition du corps médical à travers le pays, la mobilisation des parents de patients via des associations, la stimulation de la recherche dans les facultés libanaises, et la formation de personnel capable d'assurer une prise en charge psychique.

\section{LIENS D'INTÉRÊT}

Les auteurs déclarent n'avoir aucun lien d'intérêt concernant les données publiées dans cet article.

\section{ÉCHANGES AVEC LA SALLE}

\section{Pierre-Charles Batoche}

Je suppose qu'en France, de très nombreux enfants d'origine algérienne et atteints de la mucoviscidose ont été testés. Avez-vous accès à leurs mutations?

\section{Rachida Boukari}

Sur le registre mucoviscidose de Lyon, près de $10 \%$ des patients testés sont d'origine maghrébine. Les mutations de ces patients montrent que la mutation delta F 508 est la plus fréquente (à hauteur de $20 \%$ ). Des mutations très rares en France existent en Algérie. Si nous pouvions connaître, via les données 2011 du registre de la mucoviscidose, le nombre de patients maghrébins et la répartition précise des mutations, ce serait très appréciable. Pour autant, ces données ne nous feront pas faire l'économie de tests dans la population actuelle en Algérie. Leurs résultats sont d'autant plus importants que de nouvelles molécules apparaissent, pour des mutations rares, mais aussi la mutation delta F 508. Ainsi ce génotypage présente toujours un réel intérêt.

\section{De la salle}

Quels sont les sujets prioritaires pour faire du « réseautage » maladies rares autour de la Méditerranée?

\section{Marie-Françoise Bendridi}

II serait intéressant d'une part de réunir l'ensemble des laboratoires qui réalisent des confirmations diagnostiques, et d'autre part de s'interroger sur les financements de ce réseautage. Lorsque l'on demande un diagnostic anténatal pour une maladie métabolique, des moyens financiers sont nécessaires. Le réseautage pourrait contribuer au financement des confirmations diagnostiques des maladies traitables ou des maladies pour lesquelles un conseil génétique peut être donné. Dans certains pays, le traitement ne peut pas être financé. Cela pose un vrai problème éthique. Les compétences à développer sur place devraient également être intégrées dans ce réseautage.

\section{Hicham Mansour}

Une partie de ce réseau est d'ores et déjà constitué par Orphanet. Cet outil, accessible à tous, m'est très utile, notamment pour savoir dans quel laboratoire envoyer un patient atteint d'une maladie rare. La prise en charge financière des examens reste problématique. Un patient libanais ne peut pas payer 2000 \$ (soit trois mois d'un salaire moyen) pour un examen.

\section{Brigitte Chabrol}

L'accès au diagnostic et le dépistage sont contraints par la politique nationale de santé. Quelles relations pouvez-vous avoir, en tant que professionnels de santé, avec les systèmes de santé de vos pays? Comment faire émerger l'importance des maladies « rares »-ce qualificatif est à relativiser, compte tenu du taux de consanguinité dans certaines régions du Maghreb?

\section{Rachida Boukari}

Le dépistage néonatal est une urgence dans les pays du Maghreb. Ce sujet prioritaire est mentionné depuis plus de 30 ans. Je ne pose pas le problème en termes de financement, mais en termes de partage d'expérience. Ainsi nous pourrons gagner du temps et procéder directement aux dépistages. Tant que nous ne serons pas en mesure de communiquer des chiffres précis à nos décideurs, ils ne s'occuperont pas des maladies rares. Comme il nous revient d'apporter des arguments, la constitution d'un registre national est essentielle.

\section{Marie-Françoise Bendridi}

Nous avons réalisé un travail avec le concours de tous les centres des associations françaises et belges de dépistage, pour la PCU. Tous les enfants à patronyme musulman ont été répertoriés. Selon nos calculs, l'incidence de la phénylcétonurie serait de 1/3600 en Tunisie. $\varepsilon$ n outre, nous avons testé le dépistage dans les maternités.

\section{Hicham Mansour}

En matière de dépistage néonatal, il ne faut pas hésiter à utiliser les médias. Il y a six ans au Liban, nous 
dépistions seulement la phénylcétonurie, l'hypothyroïdie et la galactosémie. Ensuite deux laboratoires ont été en mesure de réaliser le dépistage des maladies métaboliques. Après quelques reportages diffusés à la télévision, des parents sont venus demander cet examen. Désormais, les patients qui n'ont pas eu un bon dépistage à la naissance sont très rares. Quand les moyens financiers sont limités, le recours aux médias est très utile.

\section{Gilles Roche, Eurobiomed}

La participation à des essais cliniques internationaux, notamment avec la France, permet à des patients d'être pris en charge gratuitement et de bénéficier d'investissements pour du matériel de diagnostic. Menez-vous une politique ferme dans ce domaine?

\section{Marie-Françoise Bendridi}

Nous faisons des essais cliniques depuis 2008. Il serait vraiment appréciable que du matériel nous soit envoyé. En général, nous effectuons l'envoi de prélèvements - bien que ceux-ci puissent parfois être utilisés à diverses fins à l'étranger. Par ailleurs, la réalisation d'essais cliniques dans le cadre d'un contrat écrit est intéressante, car celui-ci peut stipuler que les malades seront traités jusqu'à la commercialisation du produit en Tunisie. Initialement, ce dispositif était apprécié, car les firmes avaient besoin de patients. Lorsqu'elles veulent commencer à négocier, elles cherchent à détailler les conditions de réalisation d'essais cliniques dans une lettre à part, et non dans un contrat. En outre, elles précisent dans cette lettre qu'elles sont «disposées » à traiter les patients jusqu'à la commercialisation du produit, mais elles ne s'y engagent pas. Les contrats encadrant les essais cliniques réalisés dans nos pays doivent être clairs pour éviter les déconvenues. 